\title{
Effects of Emerita brasiliensis Flour Supplementation on Normotensive (Wistar) and Spontaneously Hypertensive Rats (SHR)
}

\author{
Victor Agati Cavargere, Camille Feitoza França, Ricardo Cardoso and Lucia Marques \\ Vianna* \\ Laboratório de Investigação em Nutrição e Doenças Crônico-degenerativas; Universidade Federal do Estado do \\ Rio de Janeiro; Rua Dr Xavier Sigaud 290, Urca; 22240-040; Rio de Janeiro - RJ - Brasil
}

\begin{abstract}
Several studies characterize the connection of overweight and obesity with chronic diseases. Therefore, new alternatives are being studied for controlling hypertension, such as chitin and chitosan fibers, commonly found on crustacean's carapace like Emerita brasiliensis. Rats from two different strains were divided into control and supplemented groups $(n=6)$. The Wistar strain experiment started with a 14-days baseline period, followed by supplementation of E.brasiliensis flour added to the diet in the doses of 5, 10 and 20\%, for a 14-days period each. With the optimal dose of 20\%, the study was undertaken with SHR rats, starting with a 7-days baseline period, followed by three weeks of supplementation. Data were evaluated using one-way ANOVA and p<0.05 was significant. There was a clinical reduction of systolic blood pressure on Wistar rats, but only SHR rats showed a significant reduction of systolicblood pressure and total cholesterol, LDL-cho with an increase of HDL-cho. Those findings were not related to body weight changes but, instead they were associated to E. brasiliensis flour effect on serum lipid profile. This study showed that this flour could be an alternative for controlling the hypertension and hypercholesterolemia.
\end{abstract}

Key words: chitin, chitosan, Emerita brasiliensis, blood pressure

\section{INTRODUCTION}

The chronic diseases are among the major public health problems in developing countries, and are usually associated with overweight and obesity (Ausar et al. 2003). Thus, new alternatives of controlling body weight are being studied in order to reduce the prevalence of these diseases, including hypertension, which currently affects 15 to $20 \%$ of the adult population over 18 years and reaches 50\% in elderly (Duncan et al. 1993).
Because it is an asymptomatic disease, the hypertensive patient may, abruptly, have a stroke or a fatal heart attack (Mahan and Escott- Stump 2005).

Given this, new alternative therapies to treat the hypertension and related diseases (França et al. 2010) have encouraged studies on the possible effects of the biopolymer chitin-chitosan, commonly found in the carapace of crustaceans such as Emerita brasiliensis, in reducing the blood pressure (Kanauchi et al. 1994). Another important

*Author for correspondence: lindcd@ig.com.br 
factor for the use of this animal is the fact that it is easily found throughout the Brazilian coast, being quite consumed by the local population. Chitin and chitosan polymers are nontoxic, biodegradable, biocompatible and produced by the renewable natural sources, whose properties have been used in industrial applications and technology for almost seventy years (Roberts 1992; Goosen 1996). Their structures are composed by the polymers of 2-acetamido-2-deoxy-Dglucopyranose and 2-amino-2-deoxy-Dglucopyranose, respectively, joined by glycosidic $\beta$ (1-4) bindings. It's performances in recent studies show that, once ingested, this substance is solubilized in the stomach forming a chemically active gel, because of the amino groups present in its structure. This gel has the ability to attract and bind to lipids of the diet forming a complex chitinchitosan-lipid, preventing the action of lipases, and, consequently, their absorption by the body (Wang et al. 2009).

Furthermore, chitosan, through it's free amino groups, has the ability to bind bile acids in the digestive system excreting them in the stool. Bile acids, substances present in the bile, are essential to the digestive process. They are reused by the body after the digestive process by re-absorption through the intestinal tract, returning to the liver and, thus, completing a cycle in the body, called the enterohepatic circulation (Bhattarai et al. 2009). However, the excessive presence of these acids in the intestine is related to the development of some cancers such as colon and prostate (Gallaher et al. 2000). When chitosan binds to bile acids in excess, it plays a dual beneficial effect: avoids the presence of these free acids in the intestine preventing the cancer and, simultaneously, stimulates the liver to produce more bile acids, lowering the LDL cholesterol levels in the body (Jull et al. 2005). Therefore, chitosan shows itself as an alternative of great importance in the prevention of cardiovascular diseases, because LDL cholesterol is responsible for the formation of atherosclerotic plaques, which contributes from a simple increase in the blood pressure to stroke (Jull et al. 2005).

With these potential benefits, there was an increase in the consumption of products with this fiber, especially by the people looking to reduce the body weight and to control the blood pressure. However, studies are still scarce and the data are controversial (Mhurchu et al. 2004).

\section{MATERIALS AND METHODS}

\begin{abstract}
Animals
Wistar rats (normotensive model) and SHR rats (spontaneously hypertensive model), at eight weeks of age, were obtained from the colony of bioterium of the Federal University of the State of Rio de Janeiro, and divided into control and supplemented with Emerita brasiliensis flour groups $(n=6)$. The animals were kept in the cages in individual metabolic control conditions: temperature $\left(21 \pm 2{ }^{\circ} \mathrm{C}\right)$, humidity $(60 \pm 10 \%)$, air exhaustion cycle $(15 \mathrm{~min} / \mathrm{h})$ and $12 \mathrm{~h}$-dark/light cycle (artificial lights, 7 a.m. -7 p.m.) and fed a standard diet Nuvilab (Nuvital®) plus water $a d$ libitum. All the procedures were carried out in accordance with the conventional guidelines for the experimentation with the animals (NIH Publication No. 85-23, revised 1996). The experimental protocols used in this study were approved by the Ethics Committee for Animal Experimentation at the Federal University of Rio de Janeiro State.
\end{abstract}

\section{Physiological parameters}

The animals maintained in the metabolic cages were submitted to a daily evaluation of water and food intake, body weight, diuresis and physical aspects: distribution and coloring of hair; bleeding, stains, cracks, opacification and colouring of mucous. The behavioral aspects and motorsensory parameters were also investigated following Whishaw and Kolb methodology (Whishaw and Kolb 2005). Systolic blood pressure was determined through non-invasive method of plethysmography (Vianna et al. 1992).

\section{Sample collection and preparation}

The collection of the crustaceans was held at the Reserve Beach, in Barra da Tijuca (Rio de Janeiro) in the morning, between 8 a.m. and 10 a.m. Then, the sample was prepared by the following steps: animal cleaning to remove the sand and impurities, animal storage at $-20^{\circ} \mathrm{C}$, flour making by crushing crustaceans and, finally, the mixture of theflour with standard animal diet at previously established doses.

\section{Supplementation}

After 14 days of baseline period, six rats of the normotensive strain received supplementation of 
E. brasiliensis flour, added to standard diet, in concentrations of 5, 10 and $20 \%$, for two weeks for each concentration, to define the optimal dose. When compared to the control group of this same strain, only the a $20 \mathrm{~g} / 100 \mathrm{~g}$ dose showed a significant difference. Hence, in the hypertensive strain experiment, this dose was considered as optimal and used for three weeks, following a seven days baseline period.

\section{Lipidic Profile}

The total cholesterol, HDL-cholesterol and triglycerides were analysed by the enzymatic assay. The levels of LDL-cholesterol were calculated using the Friedewald formula: $\mathrm{LDL}=$ TC - HDL - (TG / 5).

\section{Statistical Analysis}

Data were evaluated using the statistical test of variance one-way ANOVA, and $\mathrm{p}<0.05$ was considered significant.

\section{RESULTS}

The treatment did not alter the overall biological, physical and sensory motor parameters of normotensive Wistar or SHR rats (Table 1). Wistar rats presented a reduction of total cholesterol and LDL fraction; however, HDL-cholesterol had a small increase which was not statistically significant $\quad(p<0.068) \quad($ Table 2$) . \quad$ The supplementation did not alter the stool consistency or color in both the strains.

The systolic blood pressure of Wistar rats was not statistically affected by the supplementation $(p<0.077)$ (Fig. 1). On theother hand, SHR strain presented a statistically significant reduction $(p<0.05)$ of systolic blood pressure during the second week of supplementation, with $195.4 \mathrm{mmHg}$ versus $208.6 \mathrm{mmHg}$ in the control group. At the last week, the reduction remained significant with values of $193.6 \mathrm{mmHg}$ in the supplemented group versus $209.2 \mathrm{mmHg}$ in the control group (Fig. 2). Regarding the lipid profile, results showed a significant reduction $(\mathrm{p}<0.05)$ in total cholesterol and LDL-cholesterol, as well as an increase in HDL-cholesterol (Table 3).

Table 1 - Effects of Emerita brasiliensis flour on biological parameters of Wistar and SHR rats. The values represent the mean \pm SD of 6 animals (control) and 6 animals (treated) for both group.

\begin{tabular}{cccccc}
\hline Groups & Paramaters & Body Weight $(\mathbf{g})$ & Diuresis $(\mathbf{m L})$ & Food intake $(\mathbf{g})$ & Water intake $(\mathbf{m L})$ \\
\hline Wistar & Control & $308 \pm 19,15$ & $4,64 \pm 1,23$ & $19,67 \pm 2,03$ & $28,53 \pm 1,25$ \\
Rats & Treated & $295,8 \pm 21,3$ & $4,17 \pm 1,82$ & $22,07 \pm 1,07$ & $27,12 \pm 1,04$ \\
\hline SHR & Control & $239,04 \pm 1,45$ & $4,94 \pm 0,49$ & $22,15 \pm 2,58$ & $30,5 \pm 5,54$ \\
Rats & Treated & $231,48 \pm 1,92$ & $4,68 \pm 2,41$ & $21,09 \pm 3,04$ & $29,97 \pm 4,45$ \\
\hline
\end{tabular}

Table 2 - Effect of Emerita brasiliensis flour supplementation on lipidic profile in Wistar group according to the dose after 4 weeks of the experiment. These values represent the mean \pm SD of 6 control and supplemented rats $\underline{(\mathrm{p}<0.05) \text {. }}$

\begin{tabular}{ccc}
\hline Lipidic Profile & Control Group $(\mathbf{n = 6})$ & Treated Group $(\mathbf{n}=\mathbf{6})$ \\
\hline Total Cholesterol (mg/dL) & $48,5 \pm 13,5$ & $37,5 \pm 16,5$ \\
LDL-Cholesterol (mg/dL) & $17,2 \pm 1,1$ & $7,9 \pm 2,2$ \\
HDL-Cholesterol (mg/dL) & $8,4 \pm 3,0$ & $10,7 \pm 3,2$ \\
\hline
\end{tabular}

Table 3 - Effect of Emerita brasiliensis flour supplementation at a dose of 20g/100g in the lipidic profile of the SHR group after 4 weeks of the experiment. These values represent the mean \pm SD of 6 control and supplemented rats $(\mathrm{p}<0.05)$.

\begin{tabular}{ccc}
\hline Lipidic Profile & Control Group $(\mathbf{n = 6})$ & Treated Group $(\mathbf{n = 6})$ \\
\hline Total Cholesterol $(\mathrm{mg} / \mathrm{dL}))$ & $53,2 \pm 17,2$ & $36,4 \pm 6,4$ \\
LDL-Cholesterol (mg/dL) & $9,4 \pm 2,8$ & $6,7 \pm 1,2$ \\
HDL-Cholesterol (mg/dL) & $12,4 \pm 2,4$ & $14,4 \pm 3,7$ \\
\hline
\end{tabular}




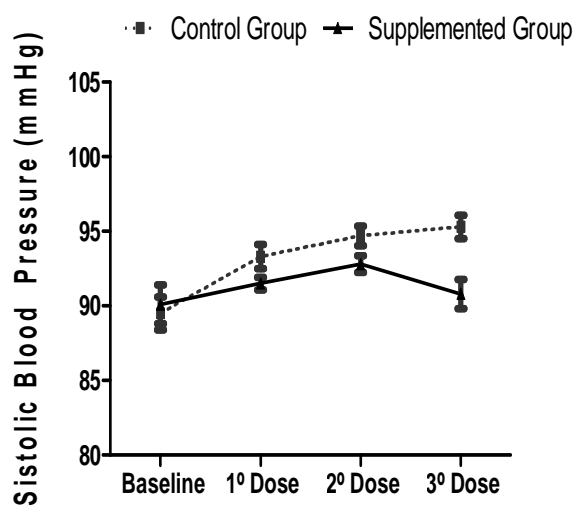

Figure 1 - Effect of supplementation of Emerita brasiliensis flour on systolic blood pressure $(\mathrm{mmHg})$ in Wistar group according to the doses. These values represent the means \pm SD of 6 control and supplemented rats $(\mathrm{p}<0.077)$.

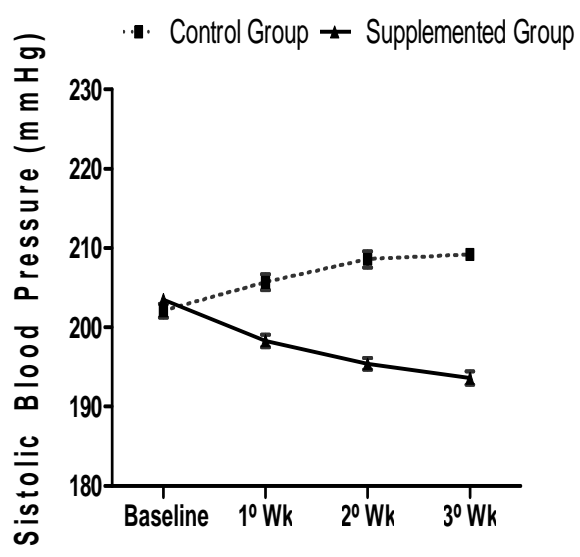

Figure 2 - Effects of supplementation of Emerita brasiliensis at dose of $20 \mathrm{~g} / 100 \mathrm{~g}$ of diet on systolic blood pressure $(\mathrm{mmHg})$ in SHR rats during the four weeks of the experiment. These values represent the means \pm SD of 6 control and supplemented rats $(\mathrm{p}<0.05)$.

\section{DISCUSSION}

This study presents new data for E. brasiliensis, which, as mentioned earlier, is regularly part of the Brazilian coastal population diet. Results for SHR rats showed a significant reduction in blood pressure starting at the second week of supplementation, while the group of normotensive rat strain (Wistar) showed only a trend in the modulation of that parameter. Since the shell of this animal contained significant amounts of chitin-chitosan biopolymer, it could be suggested that this reduction in blood pressure could reflect the ability of this compound to adsorb the lipids in the gut (Kanauchi et al. 1994).

Some studies have suggested there might be a reduction in blood pressure due to increased intake of chitosan, a positive high-density polymer that could attract and bind to the lipids (Ylitalo et al. 2002; Gallaher et al. 2002). In an acidic environment like the stomach, chitosan adsorbs fats during the digestion, facilitating their elimination through the feces. Therefore, chitosan is considered as an aid in controlling the excess fat diets. Depending on the conditions of the environment and the degree of deacetylation of chitosan (percentage of amino groups present in the biopolymer), it can hold four to five times its weight fat (Muzzarelli 1999). In sun, the favorable hypotensive effect of $E$ brasiliensis flour, here presented, was not associated to rat body weight; instead it might be related to its action on lipid profile modulation. In this study, the natural form 
of E. brasiliensis was used. However, the coastal population uses this crustacean in fried culinary preparations or cooked in risotto, both subjecting the product to heat, which prevents from stating that the effects found in this study can be extrapolated to such eating habits. Moreover, it is clear that the evaluated product, i.e, E brasiliensis flour, allows other forms of consumption of this crustacean in routine culinary preparations or even large-scale industrial enrichment of food products in general.

In conclusion, $E$ brasiliensis flour presented itself as a possible alternative therapy in the prevention and even treatment of hypertension and hypercholesterolemia.

\section{ACKNOWLEDGMENTS}

This study was supported by the Laboratory of Nutritional Investigation and DegenerativeChronic Diseases (LINDCD).

\section{REFERENCES}

Ausar SF, Morcillo M, León AE, Ribotta PD, Masih R, Vilaro Mainero M, et al. Improvement of HDL and LDL-cholesterol levels in diabetic subjects by feeding bread containing chitosan. J Med Food. 2003; 6(4):397-9.

Bhattarai N, Gunn J, Zhang M. Chitosan-based hydrogels for controlled, localized drug delivery. $A d v$ Drug Deliv Rev. 2009.

Duncan BB, Schmidt IM, Achutti AP, Polanczyk CA, Benia LR, Maia AA. Socioeconomic distribuition of noncommunicable disease risk factors in urban Brazil: the case of Porto Alegre. Bull PAHO.1993; 27:33749.

França CF, Vianna LM. The response of young and adult rats to the riboflavin supplementation. Braz. arch. biol. technol. 2010; 53(4):855-860.

Gallaher CM, Munion J, Hesslink R, Jr., Wise J, Gallaher DD. Cholesterol reduction by glucomannan and chitosan is mediated by changes in cholesterol absorption and bile acid and fat excretion in rats. $J$ Nutr. 2000; 130(11):2753-9.

Gallaher DD, Gallaher CM, Mahrt GJ, Carr TP, Hollingshead CH, Hesslink R Jr, Wise J. A glucomannan and chitosan fiber supplement decreases plasma cholesterol and increases cholesterol excretion in overweight normocholes-terolemic humans. American College of Nutr. 2002; 21(5): 428-433.
Goosen, M. E. A. Applications of chitin and chitosan. 1sr ed. Lancaster: Technomic Publishing Company; 1996.

H. Backer, R.J. Lindsey. The laboratory rat- Research application. Vol. I, II. New York: Academic Press, NRC; 1980.

Ian Q. Whishaw; Brayan Kob. The behavior of the laboratory rat- a handbook with tests. 1st Ed. Oxford: University Press Inc; 2005.

Jull AB, Ni Mhurchu C, Bennett DA, Dunshea-Mooij CAE, Rodgers A. Chitosan for overweight or obesity. Cochrane Database Syst Rev. 2005; 20(3):CD003892.

Kanauchi O, Deuchi K, Imasato Y, Koboyahi E. Increasing effects of a chitosan and ascorbica acid mixture on fecal dietary fat excretion. Applied Research Center. 1994; 7: 370-12.

Mahan LK; Escott- Stump MA. Alimentos, Nutrição e Dietoterapia. São Paulo: Editora Roca; 2005

Mhurchu CN, Poppitt SD, McGill AT, Leahy FE, Bennett DA, Lin RB, et al. The effect of the dietary supplement, chitosan, on body weight: a randomized controlled trial in 250 overweight and obese adults. Int J Obes Relat Metab Disord. 2004; 28(9): 1149-56.

Muzzarelli RA. Clinical and biochemical evaluation of chitosan for hypercholesterolemia and overweight control. EXS. 1999; 87:293-304.

Roberts, G. A. F. Chitin Chemistry. London: The Macmillan Press; 1992

Vianna LM. Efeito de administração crônica de vitamina D3 em ratos espontaneamente hipertensos. São Paulo: Escola Paulista de Medicina;1992.

Wang Y, Tu S, Li R, Yang X, Liu L, Zhang Q. Cholesterol succinyl chitosan anchored liposomes: Preparation, characterization, physical stability and drug release behavior. Nanomedicine. 2009; 13:45768.

Whishaw IQ, Kolb Bryan. The behaviour of the laboratory rat a hand book with test. Oxford: BW Oxford Academic. 2005; 68, 504-509.

Ylitalo R, Lehtinen S, Wuolijoki E, Ylitalo P, Lehtimäki T. Cholesterol-lowering properties and safety of chitosan. Arzneimitteiforschung. 2002; 52(1):1-7. 


\section{PAGINA EM BRANCO}

